\title{
Flat Frequency Comb Generation Based on Efficiently Multiple Four-Wave Mixing Without Polarization Control
}

\author{
Qimeng DONG, Bao SUN*, Fushen CHEN, and Jun JIANG
}

Key Laboratory of Optical Fiber Sensing \& Communications (Ministry of Education), University of Electronic Science and Technology of China, Chengdu, 611731, China

*Corresponding author: Bao SUN_Ｅ-mail:27450996@qq.com

\begin{abstract}
This paper presents a new technique for flat optical frequency comb (OFC) generation, which is based on the nonlinear process of multiple four-wave mixing (FWM) effects. The nonlinear effects are significantly enhanced by using the proposed optical feedback scheme consisting of a single mode fiber (SMF), two highly nonlinear fibers (HNLFs) with different zero dispersion wavelengths (ZDWs) and polarization beam splitters (PBSs). Simulation results illustrate its efficiency and applicability of expanding a comb to 128 coherent lines spaced by only $20 \mathrm{GHz}$ within 6-dB power deviation.
\end{abstract}

Keywords: Nonlinear fiber optics, multiple four-wave mixing, optical feedback, phase modulation, self-phase modulation

Citation: Qimeng DONG, Bao SUN, Fushen CHEN, and Jun JIANG, "Flat Frequency Comb Generation Based on Efficiently Multiple Four-Wave Mixing Without Polarization Control,” Photonic Sensors, 2016, 6(1): 85-89.

\section{Introduction}

The optical frequency comb (OFC) is a spectrum that consists of a set of evenly spaced frequency components with a coherent and stable phase relationship [1]. With the rapid development of the optical communication technology, OFC generation is attracting lots of interests due to its wide applications, such as the metrology [2], pulse train generation at $\mathrm{THz}$ repetition rate [3], sources for dense wavelength division multiplexing (DWDM) systems [4], multicasting in WDM passive optical networks [5], and microwave channelized receiver based on an OFC [6].

Recently, several improved schemes to flatten and broaden the OFC were reported, including a new method to efficiently generate broadband by cascaded four-wave mixing (FWM) based on launching two strong pump waves near the zero dispersion wavelength (ZDW) of an optical fiber [7], an optimized technique to generate the OFC by FWM in highly nonlinear low-dispersion fibers [8], a model with cascaded FWM and self-phase modulation (SPM) effects simultaneously occurring in the highly nonlinear fiber (HNLF) which obtained a $10 \mathrm{GHz}$ OFC of $143 \mathrm{comb}$ lines within $4.5 \mathrm{~dB}$ power deviation [9], and an expanded OFC based on the nonlinear process of multiple FWM [10] effects.

In this paper, a scheme of flat OFC generation via an optical feedback structure, consisting of two HNLFs with different ZDWs and a polarization beam splitter (PBS), is proposed. Simulation validates that the number of multiple FWM products and the power of most multiple FWM products can

Received: 18 August 2015 / Revised: 8 September 2015

(C) The Author(s) 2015. This article is published with open access at Springerlink.com

DOI: $10.1007 / \mathrm{s} 13320-015-0273-9$

Article type: Regular 
be increased by using this scheme.

\section{Theoretically analysis of the proposed scheme}

FWM effect in HNLF is a parametric process involving four different optical waves. For a typical FWM configuration, when two-pump waves at frequencies $\omega_{1}$ and $\omega_{2}$ (assume $\omega_{1}<\omega_{2}$ ) are injected into HNLF [2], the signal (at $\omega_{3}$ ) will be amplified, and the idle light (at $\omega_{4}$ ) will be generated, as shown in Fig. 1. The relationship among four light frequencies can be described with equation

$$
\omega_{1}+\omega_{2}=\omega_{3}+\omega_{4} .
$$

The momentum conservation (namely phase matching) condition should also be satisfied in the FWM process. The phase matching condition is met when the net wave vector mismatch is $\kappa=0$, where $\kappa$ can be written as [11]

$$
\begin{gathered}
\kappa=\Delta \kappa+\Delta \kappa_{N L} \\
\Delta \kappa_{N L}=\gamma *\left(P_{1}+P_{2}\right)
\end{gathered}
$$

where $\Delta \kappa$ and $\Delta \kappa_{\mathrm{NL}}$ represent wave vectors mismatches related to dispersion and nonlinear effects, respectively. $\gamma$ is the nonlinear coefficient of HNLF, and $P_{1}$ and $P_{2}$ are incident powers of $\omega_{1}$ and $\omega_{2}$, respectively. To obtain the phase matching condition, the pump wavelength should exceed the ZDW of HNLF (namely $\Delta \kappa<0$ ), so that the net wave vector mismatch can be $\kappa=0$.

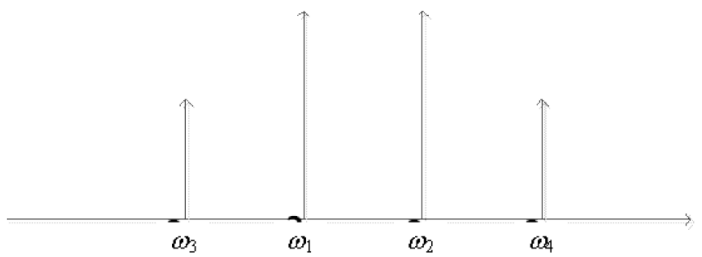

Fig. 1 Frequencies relationship of a typical FWM process.

The proposed scheme is shown in Fig. 2. Two-pump waves pass through the phase modulator to suppress stimulated Brillouin scattering. An SMF is used to compensate the chirp induced by the phase modulator. The optimal length of the SMF is about $1 \mathrm{~km}$ [9], when the modulation speed is $20 \mathrm{GHz}$. Thus, a pulse train with very short pulse-width after the SMF can be achieved. The pulse trains are then amplified by an erbium-doped fiber amplifier (EDFA). An optical filter is used to suppress the spontaneous emission noise from the EDFA. Hence, pump waves with the higher peak power are obtained, which can enhance the multiple FWM effects in the HNLF2. In such a case, the SPM effect occurs in HNLF1 simultaneously due to the optical pulse injection. The SPM effect always leads to spectrum broadening. The feedback system containing two HNLFs aims at enhancing multiple FWM effects in HNLF2 and SPM effect in HNLF1 to generate $\mathrm{OFC}$.

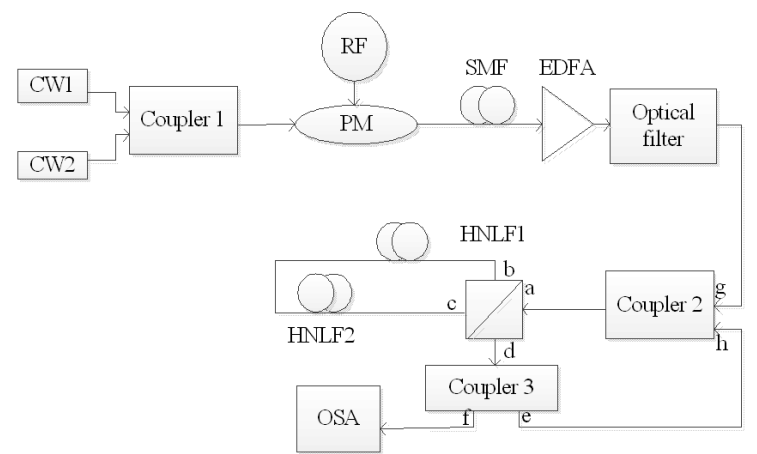

Fig. 2 Proposed OFC generator (CW: continuous wave; RF: radio frequency; PM: phase modulation; SMF: single mode fiber; EDFA: erbium doped fiber amplifier; HNLF: high nonlinear fiber; OSA: optical spectrum analyzer).

In the feedback structure, the output from Port $d$ of the PBS is fed back to the input port of Coupler 2. The system and mechanism are similar to the optical parametric oscillator [12] (OPO), which is usually used for optical amplification with a single pump. Two couplers are used for optical feeding back. The feedback ratio can be changed by using optical couplers with different coupling ratios. For example, if the coupling ratios of Couplers 2 and 3 are both 90:10 (namely Port $\mathrm{h}$ stands for 10\%, and Port e represents $90 \%$ ), the feedback ratio will be $9 \%$. If the coupling ratios of Couplers 2 and 3 are 70:30 (namely Port h stands for 30\%, and Port e represents $90 \%$ ) and 90:10, respectively, the feedback ratio is $27 \%$. If two-pump waves are injected into the feedback system with the same frequency space and only the first-order sidebands induced by the FWM 
processes are considered through HNLF every time, the total number of optical waves inside the fiber is 4 according to this recurrence formula [13] listed by (4). After the first round trip, the total number of optical waves inside the fiber will be 10 .

$$
n=3 * N-2
$$

where $N$ represents the number of pump lights launched into the feedback system, and $n$ means the total number of optical waves inside the fiber.

The PBS makes multiple FWM effects in HNLF efficient without a polarization controller (PC).

\section{System simulation and results discussion}

Two continuous wave lasers fixed at $1553 \mathrm{~nm}$ and $1552.9 \mathrm{~nm}$ are coupled together with a $3-\mathrm{dB}$ coupler. Pulse trains with very short pulse-width after the SMF are achieved in Fig. 3. The pulse trains are then amplified to $1 \mathrm{~W}$ by the EDFA. Then, the coupling ratios of Couplers 2 and 3 are 50:50 (namely Port $\mathrm{h}$ stands for 50\%) and 90:10 (Port e represents $90 \%$ ) to constitute a feedback system according to past works [14], and the feedback ratio is $45 \%$. In the feedback system, two HNLFs with different ZDWs (the ZDW of HNLF1 is $1600 \mathrm{~nm}$, and the ZDW of HNLF2 is $1550 \mathrm{~nm}$ ) and a PBS are inserted. The length, nonlinear coefficient, and dispersion slope of the HNLFs are $L=30 \mathrm{~m}, \gamma=$ $26 \mathrm{~W}^{-1} \cdot \mathrm{km}^{-1}$, and $S_{0}=0.03 \mathrm{ps} / \mathrm{nm}^{2} / \mathrm{km}$. Here, we simulate with four different configurations. In the first configuration, we remove the SMF in the setup of Fig. 2. The output OFC is presented in Fig. 4, where about 15 coherent lines are obtained within $10-\mathrm{dB}$ power deviation. In the second configuration, we remove HNLF1 in the setup of Fig. 2. The output OFC is shown in Fig. 5 where about 25 coherent lines are obtained within 10-dB power deviation. In the third configuration, we remove Coupler 2, Coupler 3, and PBS in the setup of Fig. 2. Pump lights after the SMF are injected into two HNLFs. The output OFC of two HNLFs is obtained as following in Fig. 6 where about 16 coherent lines are obtained within 8-dB power deviation. Finally, the output OFC for the proposed scheme (namely Fig. 2) is shown in Fig. 7, where the proposed scheme produces 128 coherent lines spaced to only $20 \mathrm{GHz}$ within 6-dB power deviation. Compared with other schemes, the proposed scheme can not only increase the number of frequency lines, but also improve the flatness of it.

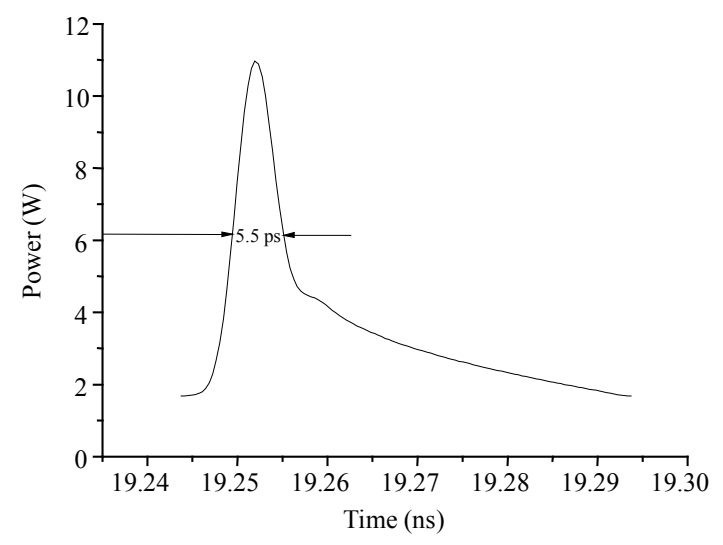

Fig. 3 Pulse shape of a pump after the SMF.

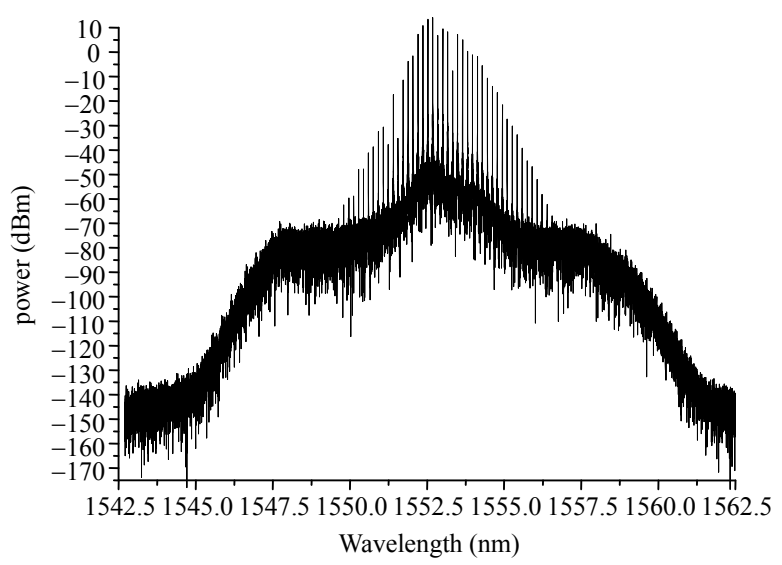

Fig. 4 Output OFC without the SMF.

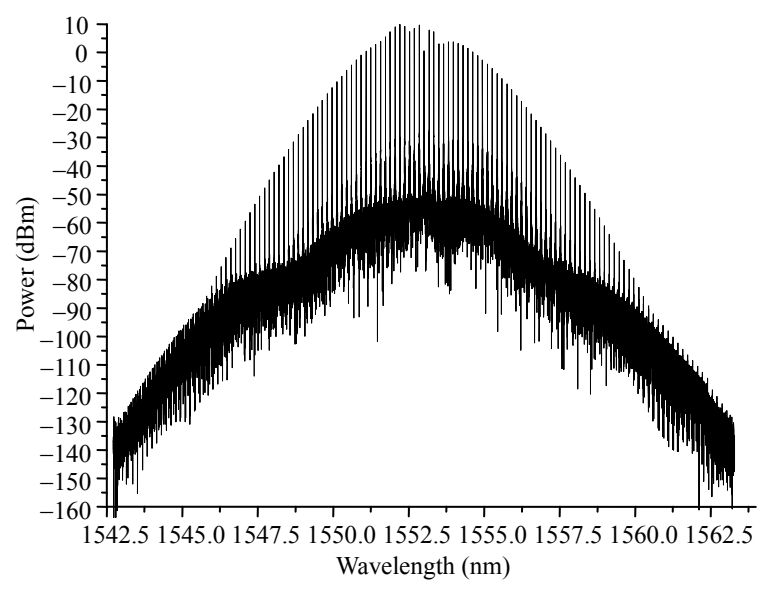

Fig. 5 Output OFC without HNLF1. 


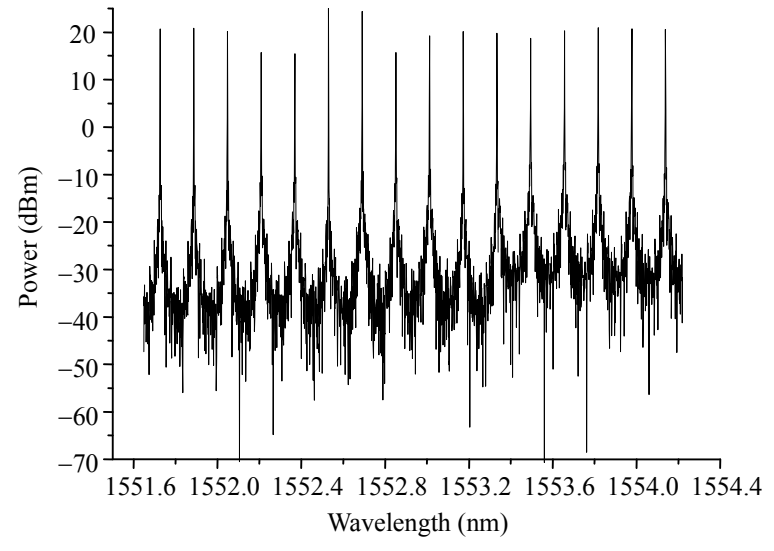

Fig. 6 Output OFC without feedback.

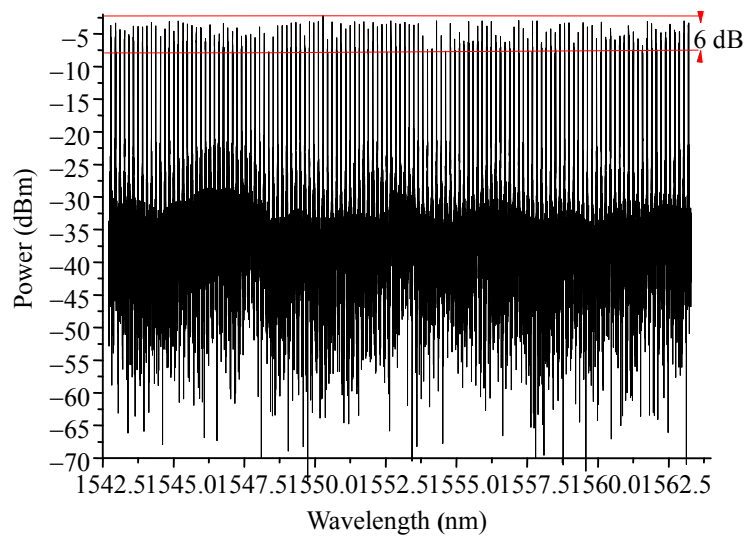

Fig. 7 Output OFC for the proposed scheme.

Finally, we investigate the impact of the output pump power of EDFA and the length of two HNLFs on the available spectral lines by the following two graphs. In Fig. 8, the length of two HNLFs is $30 \mathrm{~m}$. Other parameters are the same as the above simulation. The number of frequency lines with $\Delta P=10 \mathrm{~dB}$ (below the max power of the multiple FWM products) increases when the output power of EDFA increases. When the pump power is fixed at $0.8 \mathrm{~W}$, the number of frequency is 135 . When the output power of EDFA increases further, the number of frequency decreases. When the output power of EDFA is $1.5 \mathrm{~W}$, the number of frequency lines is 58 , which is smaller than 135 . The output power of EDFA has an optimal value. This is caused by the phase matching condition. From (3), we know that when the output power of EDFA increases, $\Delta \kappa_{\mathrm{NL}}$ becomes larger while $\Delta \kappa$ is invariable. It affects the net phase mismatching $\kappa$. When the output power of
EDFA is $0.8 \mathrm{~W}$, the phase matching is satisfied perfectly, which produces the largest number of frequency lines. Then, the output power of EDFA fixes at $0.8 \mathrm{~W}$. Other parameters are the same as the above simulation. The number of frequency lines with $\Delta P=10 \mathrm{~dB}$ related to the length of the fiber is shown in Fig. 9. Clearly, after a maximum is reached, the number of frequency with an acceptable $\Delta P$ decreases when the length of HNLFs increases. This makes clear that the use of very short fiber lengths enables highly efficient generation of the OFC. For this fiber, the optimal length is around $L=30 \mathrm{~m}$. Similarly, more frequency lines imply better phase matching. In order to ensure this phase matching, $\Delta \beta L<\pi$ [7] has to be satisfied where $\Delta \beta$ is the propagation constant mismatch.

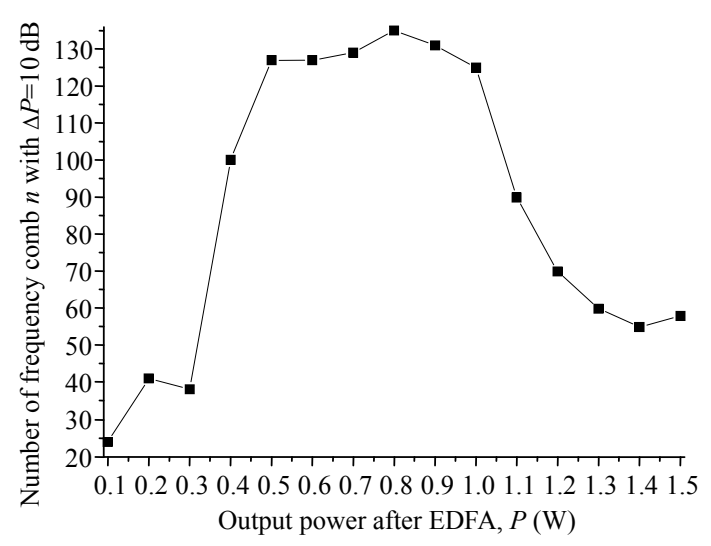

Fig. 8 Relationship between the number of frequency lines and output power of EDFA.

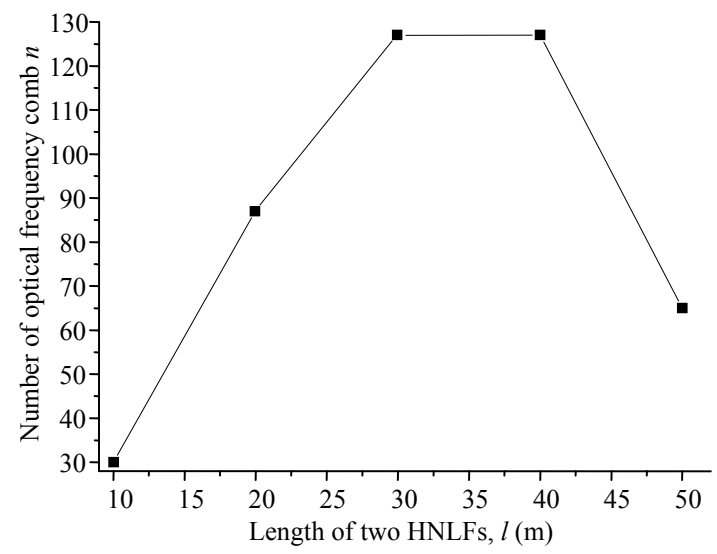

Fig. 9 Relationship between the number of frequency lines and length of two HNLFs. 


\section{Conclusions}

We propose an efficient all-optical approach to generate the OFC by simulation. An optical feedback structure including two HNLFs with different ZDWs and a PBS is used. System simulation has illustrated the efficiency of the proposed scheme of expanding a comb to 128 coherent lines spaced to only $20 \mathrm{GHz}$ within 6-dB power deviation. The optical feedback structure is used for multicasting in WDM passive optical networks. The proposed scheme does not need a PC.

\section{Acknowledgment}

This work was supported by the National Science Foundation of China (NSFC 61307088) and the Fundamental Research Funds for the Central Universities (ZYGX2012J008).

This work was supported by the National Natural Science Foundation of China (Nos. 61307088, 61205067, and 61505020) and the National Defense Pre-Research Foundation of China (9140A02050413DZ02023).

Open Access This article is distributed under the terms of the Creative Commons Attribution 4.0 International License (http:// creativecommons.org/licenses/by/4.0/), which permits unrestricted use, distribution, and reproduction in any medium, provided you give appropriate credit to the original author(s) and the source, provide a link to the Creative Commons license, and indicate if changes were made.

\section{References}

[1] T. Udem, R. Holzwarth, and T. W. Hänsch, "Optical frequency metrology," Nature, 2002, 416(6877): $233-237$.

[2] F. C. Cruz, "Optical frequency combs generated by four-wave mixing in optical fibers for astrophysical spectrometer calibration and metrology," Optics Express, 2008, 16(17): 13267- 13275.

[3] G. M. Macfarlane, A. S. Bell, E. Riis, and A. I. Ferguson, "Optical comb generator as an efficient short-pulse source," Optics Letters, 1996, 21(7): $534-536$

[4] T. Ohara, H. Takara, Y. Yamamoto, H. Masuda, T. Morioka, M. Abe, et al., "Over-1000-channel ultra-dense WDM transmission with multicarrier source," Journal of Lightwave Technology, 2006, 24(6): $2311-2317$.

[5] M. P. Fok and C. Shu, "Multi-pump four-wave mixing in a photonic crystal fiber for $6 \times 10 \mathrm{~Gb} / \mathrm{s}$ wavelength multicasting of DPSK signals," IEEE Photonics Technology Letters, 2007, 19(15): 1166- 116.

[6] Z. Li, H. Chi, X. Zhang, S. Zheng, X. Jin, and J. Yao, "A reconfigurable photonic microwave channelized receiver based on an optical comb," in 2011 International Topical Meeting on Microwave Photonics, Singapore, pp. 296-299 2011.

[7] A. C. Sodre, J. M. Boggio, A. A. Rieznik, H. E. Hernandez-Figueroa, H. L. Fragnito, and J. C. Knight "Highly efficient generation of broadband cascaded four-wave mixing products," Optics Express, 2008, 16(4): $2816-2828$.

[8] A. C. J. Sodre, J. D. Marconi, H. E. Hernandez-Figueroa, and H. L. Fragnito "Broadband cascaded four-wave mixing by using a three-pump technique in optical fibers," Optics Communications, 2009, 282(22): 4436-4439.

[9] T. Yang, J. Dong, S. Liao, D. Huang, and X. Zhang "Comparison analysis of optical frequency comb generation with nonlinear effects in highly nonlinear fibers," Optics Express, 2013, 21(7): $8508-8520$.

[10] S. A. S. Melo, A. R. do Nascimento Jr, A. C. S. Jr, L. H. H. Carvalho, D. M. Pataca, J. C. R. F. Oliveira, et al., "Frequency comb expansion based on optical feedback, highly nonlinear and erbium-doped fibers," Optics Communications, 2013, 312(1278): 287-291.

[11] G. P. Agrawal, Nonlinear fiber optics, Fourth edition. New York, U. S. A.: Academic Press, 2006: 276-278

[12] M. E. Marhic, K. K. Y. Wong, L. G. Kazovsky, and T. E. Tsai, "Continuous-wave fiber optical parametric oscillator," Optics Letters, 2002, 27(16): $1439-1441$

[13] X. Liu, "Theory and experiments for multiple four-wave-mixing processes with multi-frequency pumps in optical fibers," Physical Review A, 2008, 77(4): 043818.

[14] J. Li, X. Xiao, L. Kong, and C. Yang, "Enhancement of cascaded four-wave mixing via optical feedback," Optics Express, 2012, 20(20): 21940-21945. 